\title{
Epidemiología de la fractura de cadera en el adulto atendido en un hospital de tercer nivel
}

\author{
Diana Fernanda Caicedo Sanguino' ${ }^{1}$, Andrés Eduardo Araque Melo ${ }^{1}$ (D) , \\ Nicolás Ricardo Rojas Quintero' (D) Salvador Enrique Villamizar Quintero' \\ RESUMEN
}

Introducción: las fracturas del fémur proximal, también llamadas fracturas de cadera, se encuentran entre las principales causas de morbilidad en el mundo, donde la población más afectada son los adultos. Esto produce serias repercusiones en la salud pública, debido a su alto costo socioeconómico y la incapacidad laboral que genera.

Objetivo: determinar la epidemiología de la fractura de cadera en el adulto atendido en un hospital de tercer nivel de Cúcuta (Norte de Santander, Colombia).

Materiales y métodos: estudio descriptivo, transversal, de análisis retrospectivo, con enfoque cuantitativo. Se tomaron 175 pacientes con fractura de cadera que cumplían con los criterios de inclusión, con una población diana de mayores de 50 años de edad. Adicionalmente se registraron: edad, género, causa, comorbilidad, clasificación, intervalo de cirugía y tipo de intervención quirúrgica.

Resultados: de los 175 pacientes, se encontró una edad media general de 75,35 años. El 60\% fueron mujeres y la causa más común de la fractura correspondió a factores extrínsecos. La mayoría de los pacientes no padecía ninguna enfermedad, y en mayor proporción, acudieron por una fractura intertrocantérica. El $50 \%$ de los pacientes fueron intervenidos quirúrgicamente en un periodo superior a los 4 días.

Conclusiones: a partir de los hallazgos encontrados, se acepta que la frecuencia de la fractura de cadera está asociada con diversas variables, analizadas en este estudio, que obtuvo resultados novedosos y que quedan a disposición de la academia en bienestar de la salud, a fin de que se impulsen nuevos estudios.

Palabras clave: fracturas de cadera; epidemiologia; clasificación; comorbilidad; adulto.

1 Universidad de Santander (Cúcuta, Colombia).

Autora de correspondencia: Diana Fernanda Caicedo Sanguino. Correo electrónico: dianafernanda08@hotmail.com

Citar este artículo así:

Caicedo Sanguino DF, Araque Melo AE, Rojas Quintero NR, Villamizar Quintero SE. Epidemiología de la fractura de cadera en el adulto atendido en un hospital de tercer nivel. Rev Investig Salud Univ Boyacá. 2020;7(2):18-32. https://doi.org/10.24267/23897325.551 


\title{
Epidemiology of hip fracture in adults treated at a tertiary level hospital
}

\begin{abstract}
Introduction: Proximal femur fractures, also called hip fractures, are among the main causes of morbidity in the world where the most affected population is adults. This greatly affects public health due to its high socio-economic cost and the incapacity for work it generates.
\end{abstract}

Objective: To determine the epidemiology of hip fracture in adults treated in a tertiary hospital in Cúcuta (Norte de Santander, Colombia).

Materials and methods: A descriptive, cross-sectional study, of retrospective analysis, with a quantitative approach. 175 patients with hip fracture, who met the inclusion criteria, were taken with a target population over 50 years of age. In addition to the variables that were recorded: age, gender, cause, comorbidity, classification, surgery interval, type of surgical intervention.

Results: Of the 175 patients, a general mean age of 75.35 years was found, $60 \%$ corresponds to female patients, the most common cause is extrinsic factors, most of the patients did not suffer from any disease, in greater relevance they came for a intertrochanteric fracture. $50 \%$ of patients undergo surgery in a period of time greater than 4 days.

Conclusions: Based on the findings, it is accepted that the frequency of hip fracture is associated with various variables which were analyzed in this study, obtaining novel results that are available to the academy in health wellness, promoting new studies.

Keywords: hip fractures; epidemiology; clasification; comorbidity; adult. 


\title{
Epidemiologia de fratura do quadril em adultos tratados em um hospital (nível III)
}

\begin{abstract}
Resumo
Introdução: as fraturas do fêmur proximal, chamada também de fraturas de quadril, estão entre as principais causas de morbidade no mundo, onde a população mais acometida são os adultos. Isso pro-duz sérias repercussões na saúde pública, devido ao seu alto custo socioeconômico e à incapacidade para o trabalho que gera.
\end{abstract}

Objetivo: determinar a epidemiologia da fratura do quadril em adultos aten-didos em um hospital (nível III) em Cúcuta (Norte de Santander, Colômbia).

Materiais e métodos: estudo descritivo, transversal, análise retrospectiva, com abordagem quantitativa. Foram tomados 175 pacientes com fratura do quadril que preencheram os critérios de inclusão, com uma população-alvo com mais de 50 anos de idade. Além disso, foram registrados: idade, sexo, causa, comorbidade, clas-sificação, intervalo cirúrgico e tipo de intervenção cirúrgica.

Resultados: dos 175 pacientes, encon-trou-se uma média geral de idade de 75,35 anos. 60\% eram mulheres e a causa mais comum da fratura correspondeu a fatores extrínsecos. A maioria dos pacientes não tinha nenhuma doença e, em maior proporção, vieram por causa de uma fratura intertrocantérica. $50 \%$ dos pacientes foram operados em período superior a 4 dias.

Conclusões: com base nos achados, aceita-se que a frequência de fratura de quadril está associada a diversas variáveis, analisadas neste estudo, que obtiveram resultados inéditos e que se encontram à disposição da academia em bem-estar da saúde, para que novos estudos sejam promovidos.

Palavras-chave: fraturas do quadril; epidemiologia; classificação; comorbidade; adulto. 


\section{INTRODUCCIÓN}

Para 2015-2016, en Colombia se calculaba que había habido de 8000 a 10.000 fracturas de cadera solo en las mujeres (1). Las pacientes mayores de 65 años de edad equivalen a más del $95 \%$ de los casos de fractura de cadera. La mortalidad en la fase aguda de la población general tras una fractura de cadera suele ser inferior al $10 \%$ (2). Según la evidencia, un retraso quirúrgico mayor a 24-48 horas después del diagnóstico aumenta dos veces el riesgo de mortalidad a un año; de ahí la importancia de conocer en la población colombiana el efecto de la demora quirúrgica, que interviene en gran medida en el pronóstico o mortalidad del paciente $(3,4)$.

Cada año, en Estados Unidos ocurren aproximadamente $\mathbf{2 5 0 . 0 0 0}$ fracturas de cadera. Se dice que el $30 \%$ de estos pacientes fallece al año de la fractura de cadera y que más del $50 \%$ de ellos no podrá volver a su vida cotidiana (5-7). Tan solo un porcentaje de los pacientes recupera a los dos años una movilidad similar a la que tenía previa a la fractura $(8,9)$.

Las fracturas del fémur proximal, también llamadas fractura de cadera (FC), se clasifican respecto a su ubicación anatómica. Las del trocánter mayor y menor son raras, y las intertrocantéricas y del cuello de fémur ocurren con la misma frecuencia, en mayor proporción. Las complicaciones difieren dependiendo de su clasificación. Se sabe que las
FC intertrocantéricas se consolidan si se reducen y fijan adecuadamente, y rara vez se complican, a diferencia de la fractura de cuello de fémur, que es intracapsular y afecta el aporte vascular al fragmento proximal. Por esta razón, suelen suceder los cambios degenerativos de la cabeza femoral y la necrosis avascular. Las fracturas subtrocantéricas se asocian con altas tasas de seudoartrosis y roturas del implante, por fatiga del material, debido a que es una zona de alta tensión (10).

El adulto promedio presenta una carga de comorbilidades que condiciona una demora del procedimiento quirúrgico, lo cual es ventajoso y bien tolerado durante 12-24 horas; sin embargo, no debe permitirse un retraso excesivo, ya que una espera de más de 3 días duplica la mortalidad en el primer año posterior a la cirugía $(10,11)$. Además, el aumento de la expectativa de vida en los últimos años ha incrementado la prevalencia de enfermedades crónicas no transmisibles, como la osteoporosis (1).

Entre las principales causas de morbilidad en el mundo, la FC afecta más a los adultos, y ello repercute, en gran medida, en la salud pública, debido a su alto costo socioeconómico y a la incapacidad laboral que genera $(11,12)$. Este tipo de fractura se relaciona, en especial, con la edad y con la osteoporosis $(13,14)$. Se ha encontrado mayor incidencia de fracturas en pacientes $\geq 60$ años, donde predomina el sexo femenino, 
en comparación con el sexo masculino (15-17), y como principal factor de riesgo, la caída de su propia altura. Aproximadamente, la mitad de los pacientes sufre alteraciones en sus condiciones clínicas, funcionales, cognitivas y sociales $(18,19)$. Al existir pocos estudios epidemiológicos en $\mathrm{Co}-$ lombia y ninguno en Norte de Santander sobre prevalencia, incidencia, causas, entre otros aspectos, y dado que la mayor parte de la información de la que disponemos corresponde a estudios en población norteamericana o europea, se decidió realizar esta investigación, con la finalidad de conocer la prevalencia y el verdadero impacto de esta patología en la población nortesantandereana. Además, pretende incentivar la búsqueda de estrategias para prevenir esta entidad y resaltar la importancia de un manejo integral, que le permita retomar al adulto sus actividades cotidianas en el menor tiempo posible.

Así mismo, este estudio tuvo como propósito determinar la epidemiología de los pacientes adultos con FC atendidos en un hospital de tercer nivel, teniendo en cuenta las causas, las comorbilidades del paciente, el tiempo promedio del ingreso hasta la cirugía y el tipo de fractura con el rango de edad más común.

\section{MATERIALES Y MÉTODOS}

\section{Análisis estadístico}

Se realizó un estudio de tipo descriptivo, transversal, de análisis retrospectivo, con enfoque cuantitativo. Se utilizó el método inductivo, debido a que se va de lo particular a lo general. Cada caso se revisó de manera individual $y$, posteriormente, se sintetizó y generalizó la información. Así mismo, se empleó el método deductivo, porque se analizó y se descompuso el todo en cada una de las partes.

Métodos específicos de análisis: estadística descriptiva para organizar y presentar la información; estadística inferencial, utilizando modelos de correlación para medir grado de asociación, estimadores, pruebas de hipótesis e intervalos de confianza.

El universo son todos los pacientes con edad igual o mayor a 50 años, con FC recibidos en un hospital de tercer nivel de la ciudad de Cúcuta (Norte de Santander, Colombia), tratados en el periodo 2015-2017, que presentaron los datos completos de la historia clínica.

La población excluida fueron los pacientes reintervenidos, es decir, que tuvieron cirugías previas por FC o fractura de fémur, pacientes con historia clínica incompleta y pacientes con fracturas patológicas. 
Previa autorización, se solicitó la información sobre el número de adultos atendidos. Posteriormente, se consultó el número de intervenciones por FC y los datos generales de los pacientes tratados por esta causa. Luego, se consultaron bases de datos hemerográficas para obtener un promedio de los días de estancia hospitalaria posterior a la intervención quirúrgica por FC.

Se realizó un muestreo aleatorio simple en que todos los elementos de la población que cumplieron con las condiciones de inclusión tuvieron la misma oportunidad de ser seleccionados. El nivel de confianza es del $95 \%$ y el error máximo admisible es del $5 \%$, para tener una buena muestra, que no solo sea representativa con respecto al tamaño, sino en cuanto a las características o variable fundamental.

En aras de recolectar los datos de la investigación, se revisaron las historias clínicas de los pacientes con diagnóstico de fractura de cadera recibidos en un hospital de tercer nivel, tratados en el periodo 2015-2017, a fin de obtener así la información necesaria para cumplir los objetivos planteados con la hoja de datos, verificada por el asesor académico y metodológico.

La sistematización es la organización de los datos y la información, de manera que se obtenga el mayor provecho en el logro de los objetivos propuestos. En este sentido, se utilizó estadística descriptiva o deductiva que, además de haber permitido este requisito del proyecto, facilitó la presentación y el análisis de los datos e información suministrada por la muestra. Este proceso se realizó de manera tabular, gráfico (diferentes tipos) y analítico (diferentes medidas de tendencia central y dispersión, de posición, cuartiles y percentiles). También para el análisis de estadística inferencial o inductiva, para la obtención de conclusiones sobre la población, se utilizaron datos e información de la muestra, varios análisis con estadística paramétrica y a libre distribución.

\section{RESULTADOS}

\section{Epidemiología}

De los 175 pacientes que cumplieron con los criterios de inclusión y exclusión que ingresaron bajo el diagnóstico de FC a un hospital de tercer nivel entre el 2015 y el 2017 , el $60 \%(n=105)$ correspondió a mujeres. El intervalo de edades con mayor proporción corresponden de 69,5 a 89,5 años, con un $59 \%(n=104)$. Con una edad media general de 75,35 años (tabla 1). 
Tabla 1. Grupos de edades, en años, sexo

\begin{tabular}{ccccc}
\hline Categoría & Frecuencia & $\begin{array}{c}\text { Masculino } \\
(\%)\end{array}$ & $\begin{array}{c}\text { Femenino } \\
(\%)\end{array}$ & $\begin{array}{c}\text { Total } \\
(\%)\end{array}$ \\
\hline 49,5 a 59,5 & 20 & 8,00 & 3,43 & 11,43 \\
\hline 59,5 a 69,5 & 35 & 8,00 & 12,00 & 20,00 \\
\hline 69,5 a 79,5 & 52 & 9,71 & 20,00 & 29,71 \\
\hline 79,5 a 89,5 & 52 & 10,86 & 18,86 & 29,71 \\
\hline 89,5 a 99,5 & 16 & 3,43 & 5,71 & 9,14 \\
\hline & 175 & 40,00 & 60,00 & 99,99 \\
\hline
\end{tabular}

\section{Causas de fractura de cadera}

Las causas extrínsecas (mobiliario inestable, mala iluminación, piso resbaloso, alfombras o tapetes arrugados, escaleras inseguras, entre otras) tienen la frecuencia de ocurrencia más alta, con el $66,86 \%(n=117)$, de todos los casos contemplados en la muestra. Esta tendencia parece sobrepasar significativamente el, $22,86 \%$ restante, conformado por las causas intrínsecas (enfermedades que alteren la marcha, hipotensión arterial, trastorno visual o vestibular, cardiopatía, neuropatía periférica, fármacos, alcohol) y el 10,29\% de las causas situacionales (actividades que realiza el paciente).

\section{Comorbilidades medicas asociadas con la fractura de cadera}

El $44,57 \%$ de la muestra no refiere ninguna comorbilidad. La hipertensión arterial, la diabetes, la osteoporosis, el Alzheimer y la epilepsia constituyen las 5 primeras enfermedades que padece el paciente (tabla 2). Entre ellas, se destaca, en mayor grado, la hipertensión arterial, con $29,71 \%(n=52)$ de casos registrados; seguido de la diabetes mellitus, con $9,71 \%(n=17)$. La comorbilidad más común del sexo femenino fue la hipertensión arterial, con un $21,14 \%$; seguido de la diabetes, con un $7,43 \%$, y de la osteoporosis, con un $2,29 \%$.

\section{Intervalo de cirugía}

El intervalo de días transcurrido desde el ingreso del paciente al hospital hasta la ejecución de la cirugía obtuvo una mediana por encima de 4 días, con una gran variación, debido a que realizaron intervenciones hasta en el día 38. Los hombres se intervinieron con una media de 7 días, y las mujeres, con una media de 6 días (figura 1).

Figura 1. Tiempo de intervención quirúrgica

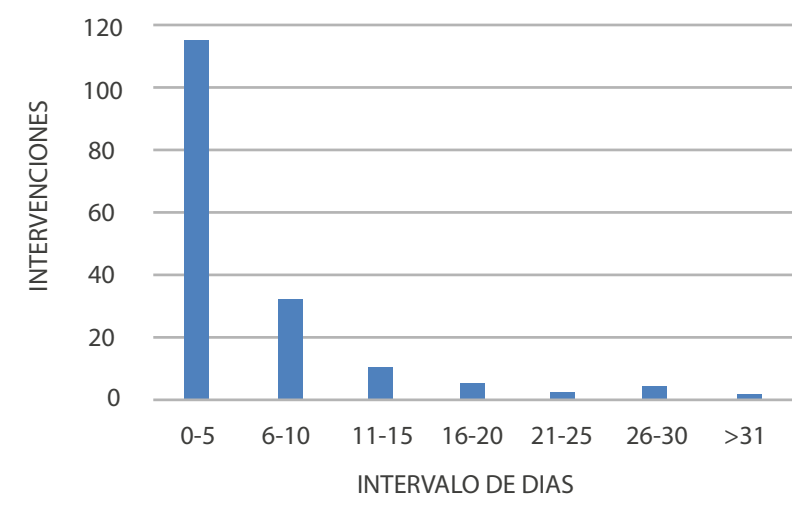


Tabla 2. Comorbilidades con la fractura de cadera

\begin{tabular}{|c|c|c|c|c|c|c|}
\hline Comorbilidad & Frecuencia masculino & $\%$ & Frecuencia femenino & $\%$ & Frecuencia total & Total (\%) \\
\hline No refiere & 37 & 21,14 & 41 & 23,43 & 78 & 44,57 \\
\hline Hipertensión arterial & 15 & 8,57 & 37 & 21,14 & 52 & 29,71 \\
\hline Diabetes & 4 & 2,29 & 13 & 7,43 & 17 & 9,71 \\
\hline Osteoporosis & 4 & 2,29 & 4 & 2,29 & 8 & 4,57 \\
\hline Alzheimer & 1 & 0,572 & 4 & 2,288 & 5 & 2,86 \\
\hline Epilepsia & 3 & 1,716 & 2 & 1,144 & 5 & 2,86 \\
\hline Enfermedad cerebro-vascular & 3 & 1,716 & 1 & 0,572 & 4 & 2,29 \\
\hline Parkinson & 1 & 0,572 & 2 & 1,114 & 3 & 1,71 \\
\hline Isquemia miocárdica & 1 & 0,572 & 2 & 1,114 & 3 & 1,71 \\
\hline Hipotiroidismo & 1 & 0,572 & 2 & 1,114 & 3 & 1,71 \\
\hline Carcinoma & 1 & 0,572 & 2 & 1,114 & 3 & 1,71 \\
\hline Retardo mental & 0 & 0 & 3 & 1,71 & 3 & 1,71 \\
\hline Cardiopatía & 1 & 0,572 & 2 & 1,114 & 3 & 1,71 \\
\hline Anemia & 2 & 1,114 & 0 & 0 & 2 & 1,14 \\
\hline Fibrilación auricular & 0 & 0 & 2 & 1,114 & 2 & 1,14 \\
\hline Infección de vías urinarias & 1 & 0,572 & 1 & 0,572 & 2 & 1,14 \\
\hline $\begin{array}{l}\text { Enfermedad pulmonar obstructiva } \\
\text { crónica }\end{array}$ & 1 & 0,572 & 1 & 0,572 & 2 & 1,14 \\
\hline Hernias & 1 & 0,572 & 1 & 0,572 & 2 & 1,14 \\
\hline Dislipidemia & 0 & 0 & 1 & 0,572 & 1 & 0,57 \\
\hline Hiperplasia prostática benigna & 1 & 0,572 & 0 & 0 & 1 & 0,57 \\
\hline Asma & 0 & 0 & 1 & 0,572 & 1 & 0,57 \\
\hline Artritis reumatoide & 0 & 0 & 1 & 0,572 & 1 & 0,57 \\
\hline Hipoacusia & 1 & 0,572 & 0 & 0 & 1 & 0,57 \\
\hline Insuficiencia renal aguda & 1 & 0,572 & 0 & 0 & 1 & 0,57 \\
\hline Vértigo & 0 & 0 & 1 & 0,572 & 1 & 0,57 \\
\hline Ateromatosis & 1 & 0,572 & 0 & 0 & 1 & 0,57 \\
\hline
\end{tabular}




\section{Clasificación de la fractura}

Para la clasificación de la fractura, según su ubicación, las de mayor ocurrencia fueron la intertrocantérica, con $61,14 \%(n=107)$, seguida de la subcapital o cuello de fémur, con $34,29 \%(n=60)$. Prácticamente estas dos categorías cubren el 95,43\% de todos los casos estudiados (tabla 3). El tipo de intervención quirúrgica de mayor proporción es osteosíntesis, con $70,29 \%(n=123)$, vs. la artroplastia, con $29,71 \%(n=52)$.

Tabla 3. Ubicación de la fractura en el paciente

\begin{tabular}{ccc}
\hline Ubicación & Frecuencia & \% \\
\hline Intertrocantérica & 107 & 61,14 \\
\hline Transcervical & 2 & 1,14 \\
\hline Subcapital o cuello de fémur & 60 & 34,29 \\
\hline Subtrocantérica & 3 & 1,71 \\
\hline Subtrocantérica + transcervical & 2 & 1,14 \\
\hline $\begin{array}{c}\text { Intertrocantérica + subcapital o } \\
\text { cuello de fémur }\end{array}$ & 1 & 0,57 \\
\hline
\end{tabular}

\section{DISCUSIÓN}

A partir de los hallazgos encontrados, se acepta que la frecuencia de la FC aumenta con la edad. Para nuestro estudio, la edad media fue de 75,35 años. Teniendo en cuenta que el $95 \%$ de los intervalos de confianza que se estimen van a contener los valores reales de la edad, en nuestro muestreo, el intervalo de confianza estuvo entre 74,82 y
78,59 años de edad. Hubo mayor presentación de FC en el sexo femenino, con 76,71 años, lo que contrasta levemente con otros estudios, como el realizado por Serra et al. (20), en España, donde de 146.383 FC, fueron estudiadas 130.414 (89\%). Estas correspondieron a mayores de 64 años, y la edad media fue de 82 años (78\% mujeres). Otro estudio de alto impacto realizado por Valdez et al. (21), en La Habana, demostró que el rango de edad de mayor incidencia fue el de los 80-90 años. La edad o rango de esta varía en diferentes poblaciones, y no hay una explicación clara para esta situación, pero probablemente se deba al estilo de vida de los pacientes, como la alimentación y el ejercicio, el cual puede influir en la presentación temprana de la FC.

En un estudio analítico de casos y controles realizado por Morales et al. (22), en el Hospital Militar Nueva Granada, con 147 pacientes, la edad promedio fue de 74,3 años (desviación estándar: 13,8 años), de los cuales el $65 \%$ eran mujeres, y el $35 \%$, hombres. El $53,6 \%$ de los pacientes presentaba hipertensión arterial, y el 15,7\%, diabetes, datos semejantes a nuestro estudio, ya que se evidenció que el sexo femenino supera con creces al masculino. Aquí el intervalo de confianza muestra que la verdadera proporción de pacientes del sexo femenino está entre el $52,74 \%$ y el $67,26 \%$. Se destacó en mayor grado la hipertensión arterial, con un $29,71 \%$ de los casos registrados; seguido de la diabetes mellitus, con un $9,71 \%$. 
No obstante, en nuestro estudio incluimos que el mayor porcentaje de los pacientes no refirió ninguna enfermedad, con un $44,57 \%$; esto probablemente por desconocimiento del propio paciente o por falta de tiempo en las urgencias para profundizar en la historia clínica, ya que la osteoporosis es frecuente en este grupo de edad. Pensamos que esta sea probablemente infradiagnosticada en nuestro medio. En el mismo estudio realizado por Morales et al. (22) se documentó que el promedio de horas entre el ingreso del paciente y la realización de la cirugía fue de 120 horas (desviación estándar: 90 horas). Entre tanto, en nuestro estudio, la mediana indica que el $50 \%$ de los pacientes tienen un intervalo de cirugía por encima de los 4 días, y el resto, por debajo de este valor, debido a la variabilidad de tiempos encontrados. De los 175 pacientes estudiados, 19 fueron intervenidos luego de 12 días; además, con rangos de 1-38 días para los hombres y 1-27 días para las mujeres.

En México, Sánchez-Hernández et al. (23) estudiaron 84 pacientes con fractura intertrocantérica, de los cuales $43(51,11 \%)$ tenían diabetes mellitus; 32 (38,09\%), hipertensión arterial sistémica; 9 $(10,71 \%)$, enfermedad isquémica del corazón, y 8 $(9,52 \%)$ insuficiencia renal crónica, lo que guarda similitud con nuestro estudio, que evidenció que la comorbilidad más frecuente es la hipertensión arterial y la diabetes mellitus.
En Málaga (España), Díaz y Navas (24) llevaron a cabo un estudio transversal con 428 pacientes, de los cuales $202(51,4 \%)$ presentaron fractura cervical, y 208 (48,6\%), trocantérica. En contraste con nuestro estudio, el tipo de fractura más frecuente fue la intertrocantérica, con $61,14 \%$; seguida de la subcapital o cuello de fémur, con $34,29 \%$. Prácticamente, estas dos categorías cubren el $95,43 \%$ de todos los casos estudiados.

El aumento de la edad se asocia con mayor prevalencia de FC, debido a alteraciones del equilibrio, y dentro de sus causas está la sarcopenia, reflejada en pérdida de fuerza muscular, aumento de la masa grasa y menor rendimiento físico, por falta de actividad. La habilidad de desplazarse está comandada por múltiples sistemas, como la visión, los reflejos propioceptivos, el aparato vestibular, el sistema nervioso periférico y el sistema músculo-esquelético (25). Además, existen factores no relacionados con el paciente. En nuestro estudio se observó que los factores extrínsecos (mobiliario inestable, mala iluminación, piso resbaloso, alfombras o tapetes arrugados, escaleras inseguras, entre otras) tienen mayor frecuencia de ocurrencia, con un $66,86 \%$ de todos los casos contemplados en la muestra.

Esta tendencia parece sobrepasar significativamente al $50 \%$ restante, conformado por las causas intrínsecas $(22,86 \%)$ y situacionales $(10,29 \%)$. Santillana-Hernández et al. (26) estudiaron a 924 
pacientes, de quienes las mujeres fueron las más prevalentes y la mayoría de sus FC ocurrieron en el hogar (62\%); entre tanto, para los hombres las FC fueron más frecuentes en la vía pública (26\%). Por otro lado, los mecanismos más referidos que provocaron la caída fueron: resbalo, en 360 pacientes (39\%); tropezado o atorado, en 248 $(27 \%)$, y pérdida del equilibrio por causas diversas, en 215 (23\%).

Los datos nombrados se vinculan con nuestro estudio, ya que los factores extrínsecos se relacionan directamente con la caída del adulto, pues, según el intervalo de confianza estimado, la proporción real de pacientes que ingresan por lesiones de cadera debido a causas extrínsecas va del $59,89 \%$ al $73,83 \%$. Respecto a la relación de la FC con el sexo, destaca la superioridad de fracturas intertrocantéricas y de cuello de fémur, con el 34,86 y el $24 \%$, en el sexo femenino; en comparación con el masculino, del $26,29 \%$ y el $10,29 \%$, respectivamente (26). Un estudio realizado por Espinosa et al. (27) en un hospital de tercer nivel en Colombia, con 478 pacientes sometidos a cirugía de cadera, evidenció que el retraso quirúrgico superior a 4 días y el índice de comorbilidad de Charlson $\geq 3$, junto con anemia $<10 \mathrm{~g} /$ dl y edad $\geq 80$ años, fueron factores asociados con mayor mortalidad. Morales Piñeiro et al. (28) demostraron un predominio del sexo femenino de $2: 1$, una media de edades de 80,8 años y una moda de 91 años, las fracturas intertrocantéricas y el lado izquierdo, en una muestra de 119 fracturas en un estudio de corte transversal realizado en Cuba.

Durante nuestro estudio se realizó una profunda investigación sobre la FC en Cúcuta, proceso en el cual no se encontró una epidemiologia clara; por ello, se realizó el presente estudio, dado que es de gran importancia conocer y aclarar todo lo que concierne a las variables epidemiológicas. En el estudio actual, encontramos que la edad y el sexo que prevalece se asemeja a la literatura mundial publicada.

\section{CONCLUSIONES}

La fractura de cadera es una patología quirúrgica relativamente común en nuestro medio. Por lo general, afecta a pacientes mayores de 60 años de edad, a causa de un traumatismo de baja energía, como lo es la caída de su propia altura. Influyen diversos factores, directa o indirectamente en la FC, entre los cuales el padecimiento de osteoporosis, diabetes y alteraciones nutricionales son las más frecuentes. Afecta más a mujeres que a hombres, con una edad aproximada de 60-70 años.

La causa más común de FC corresponde a los factores extrínsecos, por lo que se recomienda prestar especial atención a la infraestructura del hogar, teniendo en cuenta la necesidad de una 
adecuada iluminación y de superficies de apoyo sólido, mediante las cuales se puedan evitar las caídas. Con frecuencia, se asocia la FC con osteoporosis, pero en nuestro estudio se presentó un subregistro importante de este antecedente patológico. El lapso entre el ingreso y la cirugía es de cuatro días en la mitad de los casos estudiados, lo cual contrasta notablemente con la literatura nacional e internacional.

\section{AGRADECIMIENTOS}

Agradecemos al Hospital Universitario Erasmo Meoz de Cúcuta, por la información referenciada del proyecto.

\section{CONFLICTOS DE INTERESES}

No se declaran conflictos de intereses

\section{FINANCIACIÓN}

Este proyecto de investigación fue financiado por los autores de este artículo.

\section{REFERENCIAS}

1. González ID, Becerra MC, González J, Campos AT, Barbosa-Santibáñez J, Alvarado R. Fracturas de cadera: satisfacción posquirúrgica al año en adultos mayores atendidos en Méderi-Hospital Universitario Mayor, Bogotá, D.C.
Rev Cienc Salud. 2016;14(3):409-22. https:// doi.org/10.12804/revsalud14.03.2016.08

2. González-Montalvo Jl, Alarcón-Alarcón T, Gotor-Pérez P, Martín-Maestre I. ¿Es posible mejorar la atención al paciente con fractura de cadera? Aportaciones del programa Fonda. En: Valverde García JA, Sáez López $P$, Sánchez Hernández $P$, editores. 3a actualización en ortogeriatría [internet]. Ávila: Sociedad Castelanoleonesa-Cántabro-Riojana de Traumatología y Ortopedia; 2015. Disponible en: https://www.researchgate. net/profile/Juan-Gonzalez-Montalvo/publication/307560150_El_Programa_FONDA-HULP para_pacientes_con_Fractura_de_Cadera/ links/57c8799708ae9d640480e19d/El-Programa-FONDA-HULP-para-pacientes-con-Fractura-de-Cadera.pdf

3. Zamora-Navas $P$, Esteban-Peña M. Seasonality in incidence and mortality of hip fracture. Rev Esp Cir Ortop Traumatol. 2019; 63(2):132-7. https://doi.org/10.1016/j.recot.2018.05.008

4. Novoa-Parra CD, Hurtado-Cerezo J, Morales-Rodríguez J, Sanjuan-Cerveró R, Rodrigo-Pérez $\mathrm{JL}$, Lizaur-Utrilla A. Factores predictores de la mortalidad al año de pacientes mayores de 80 años operados tras fractura de cuello femoral. Rev Esp Cir Ortop Traumatol. 2019; 63(3):2028. https://doi.org/10.1016/j.recot.2018.10.007 
5. Urgell $C$, Massot Mesquida $M$, Valles Fernández R, García Vicente JA, Franzi Siso A, Muñoz Ortiz $\mathrm{L}$, et al. Adecuación del tratamiento de la osteoporosis en prevención primaria. Estudio cuantitativo y cualitativo. Aten Prim. 2018; 50(1):6-15. https://doi.org/10.1016/j. aprim.2017.01.007

6. Black DM, Rosen CJ. Clinical practice: postmenopausal osteoporosis. N Engl J Med. 2016; 374 (3):254-62. https://doi.org/10.1056/ nejmcp1513724

7. Singer $B R$, McLauchlan GJ, Robinson $C M$, Christie J. Epidemiology of fractures in 15,000 adults: the influence of age and gender. J Bone Joint Surg Br. 1998; 80(2):243-8. https:// doi.org/10.1302/0301-620x.80b2.7762

8. Izaguirre A, Delgado I, Mateo-Troncoso C, Sánchez-Nuncio HR, Sánchez-Márquez W, Luque-Ramos $A$. Rehabilitación de las fracturas de cadera: revisión sistemática. Acta Ortop Mex [internet]. 2018;32(1):28-35. Disponible en: https://www.medigraphic.com/cgi-bin/ new/resumen.cgi?IDARTICULO $=80134$

9. Barrios-Moyano A, Contreras-Mendoza EG. Frecuencia de complicaciones en pacientes mayores de 60 años con fractura de cadera. Acta Ortop Mex [internet]. 2018;32(2):65-9. Disponible en: https://www.medigraphic.com/ pdfs/ortope/or-2018/or182c.pdf
10. Canale TS, Beaty JH. Campbell cirugía ortopédica Vol. 6. Madrid: Marban; 2015.

11. Artal MM, Roca-Chacón O, Martínez-Alonso M, Serrano Godoy M, Mas-Atance J, García GR. Fractura de cadera en el paciente anciano: Factores pronóstico de mortalidad y recuperación funcional al año. Rev Esp Geriatr Gerontol. 2018;53(5):247-54. https://doi.org/10.1016/j. regg.2018.04.447

12. Dy CJ, McCollister KE, Lubarsky DA, Lane JM. An economic evaluation of a systems-based strategy to expedite surgical treatment of hip fractures. J Bone Joint Surg Am. 2011; 93(14):1326-34. https://doi.org/10.2106/ jbjs.i.01132

13. Ensrud KE, Crandall CJ. Osteoporosis. Ann Intern Med. 2017; 167(3):ITC17-ITC32. https:// doi.org/10.7326/aitc201708010

14. Nossa JM, Escobar N, Márquez D, Leal E, Cabal F, Barreto A. Aplicación de un programa multidisciplinario para el manejo de fracturas de cadera en el adulto mayor. Incidencia de comorbilidades y su impacto en la oportunidad quirúrgica. Rev Colomb Ortop Traumatol. 2016;30(3):84-9. https://doi.org/10.1016/j. rccot.2016.10.005 
15. Bengoa F, Carrasco M, Amenábar PP, Schweitzer D, Botello E, Klaber I. Optimización perioperatoria del paciente anciano con fractura osteoporótica de cadera. Rev Med Chil. 2017 Nov;145(11):1437-46. https://doi. org/10.4067/s0034-98872017001101437

16. Etxebarria-Foronda í. Incidencia de la fractura de cadera en España. Med Clin (Barc). 2015;145(11):482-4. https://doi.org/10.1016/j.medcli.2015.04.009

17. Viveros-García JC, Torres-Gutiérrez JL, Alarcón-Alarcón T, Condorhuamán-Alvarado PY, Sánchez-Rábago CJ, Gil-Garay E, et al. Fractura de cadera por fragilidad en México: ¿en dónde estamos hoy? ¿Hacia dónde queremos ir? Acta Ortop Mex [internet]. 2018; 32(6):334341 Disponible en: https://www.medigraphic. com/pdfs/ortope/or-2018/or186f.pdf

18. Departamento Administrativo Nacional de Estadística (DANE). Censo Nacional de Población y Vivienda 2018 [internet]. Disponible en: https://www.dane.gov.co/index.php/estadisticas-por-tema/demografia-y-poblacion/censo-nacional-de-poblacion-y-vivenda-2018

19. Sosa Henríquez M, Gómez de Tejada Romero MJ. Osteoporosis. Rev Clín Esp. 2016;12(16):900-8. https://doi.org/10.1016/j. med.2016.07.003
20. Serra JA, Garrido G, Vidán M, Marañón $E$, Brañas $F$, Ortiz J. Epidemiología de la fractura de cadera en ancianos en España. An Med Interna (Madrid) [internet]. 2002;19(8):9-19. Disponible en: http://scielo. isciii.es/scielo.php?script $=$ sci_arttext\&pi$\mathrm{d}=$ S0212-71992002000800002\&Ing=es

21. Hirandrés Valdés HV, Nápoles-Pérez $M, P e-$ ña-Atrio GA, Pereda-Cardoso O. Morbimortalidad de las fracturas de caderas. Rev Cuba Ortop Traumatol [internet]. 2018;32(1). Disponible en: http://www.revortopedia.sld.cu/ index.php/revortopedia/article/view/140/114

22. Morales O, Parra Hernández JD, Mateus R. Morbimortalidad posterior a fracturas intertrocantéricas de cadera: efecto del retraso en el tratamiento quirúrgico. Rev Colomb Ortop Traumatol. 2018;32(1):33-7. https://doi.org/10.1016/j.rccot.2017.07.005

23. Sánchez-Hernández E, Mejía-Rohenes LC, Benítez-Romero A. Comorbilidades en pacientes operados por fractura transtrocantérica. Rev Esp Méd Quir [internet]. 2014;19(2):145-51. Disponible en: https://www.redalyc.org/articulo.oa?id=47331518002

24. Díaz AR, Navas PZ. Factores de riesgo en fracturas de cadera trocantéricas y de cuello femoral. Rev Esp Cir Ortop Traumatol. 
2018;62(2):134-41. https://doi.org/10.1016/j.

recot.2017.09.002

25. Quintar E, Giber F. Caídas en el adulto mayor: factores de riesgo y consecuencias. Actual. Osteología [internet]. 2014;10(3):278-86. Disponible en: http://osteologia.org.ar/files/pdf/ rid39_quintar.pdf

26. Santillana-Hernandez SP, Moctezuma A, Medina-Beltrán GR, Gómez-Ortega G, Cortés González RM. Caídas en el adulto mayor: factores intrínsecos y extrínsecos. Rev Med IMSS [internet]. 2002;40(6):489-93. Disponible en: https://www.medigraphic.com/pdfs/imss/ im-2002/im026f.pdf

27. Espinosa KA, Gélvez AG, Torres LP, García MF, Peña OR. Pre-operative factors associated with increased mortality in elderly patients with a hip fracture: a cohort study in a developing country. Injury. 2018;49(6):1162-8. https:// doi.org/10.1016/j.injury.2018.04.007

28. Morales Piñeiro S, Morera Estévez L, Martínez Aparicio L, Cedré González JC, Mata Cuevas R, Gómez Sarduy A. Caracterización epidemiológica de la fractura de cadera. Acta Med Cent [internet]. 2020;14(2):193-200. Disponible en: https://www.medigraphic.com/pdfs/medicadelcentro/mec-2020/mec202f.pdf 